\title{
Une cause rare de pseudoparalysie radiale
}

\section{A Rare Cause of Pseudo-Radial Paralysis}

\author{
K. Cissokho $\cdot$ S. Gerber $\cdot$ C. Gerlier
}

Reçu le 1 avril 2019; accepté le 16 mai 2019

(C) SFMU et Lavoisier SAS 2019

\section{Introduction}

La symptomatologie distale motrice isolée du nerf radial est principalement causée par une lésion du système nerveux périphérique. Elle résulte le plus souvent d'une lésion traumatique ou d'une neuropathie de compression. Très rarement, l'atteinte motrice isolée de certains doigts peut mimer des lésions nerveuses périphériques et résulter d'une atteinte du système nerveux central. Intriqué à une anamnèse trompeuse, ce symptôme isolé rend le diagnostic final de « pseudoparalysie radiale » complexe en médecine d'urgence.

\section{Cas clinique}

Nous rapportons le cas d'un patient âgé de 50 ans pris en charge au circuit court des urgences à la suite d'un traumatisme sur la face postérieure du bras gauche contre un angle de mur dix jours auparavant. Ce patient droitier décrivait une difficulté de préhension et une difficulté à étendre ses troisième, quatrième et cinquième doigts de la main gauche, avec une attitude vicieuse indolore et spontanée de ces doigts en flexion d'installation progressive depuis le traumatisme. Ses antécédents étaient un tabagisme actif (55 paquetsannée) et une notion d'alcoolisme chronique. Il était sans emploi et ne prenait aucun traitement. L'examen neurologique retrouvait un déficit de l'extension des trois derniers doigts de la main gauche prédominant aux quatrième et cinquième rayons coté à 4 sur 5 et donnant à sa main un aspect incomplet de col de cygne (les deux premiers doigts étant

\footnotetext{
K. Cissokho · C. Gerlier $(\bowtie)$

Service des urgences, groupe hospitalier Paris-Saint-Joseph, 185, rue Raymond-Losserand, F-75014 Paris, France e-mail : camille.gerlier@gmail.com

S. Gerber

Service d'imagerie médicale, groupe hospitalier Paris-Saint-Joseph, 185, rue Raymond-Losserand, F-75014 Paris, France
}

respectés). Les extensions de la main sur l'avant-bras, de l'avant-bras sur le bras, la pronation, la supination et les réflexes ostéotendineux (dont les tricipitaux) étaient normaux. Il n'y avait pas de déficit sensitif, de stigmate cutanéo-osseux du traumatisme rapporté, de signe d'ischémie vasculaire ni de cervicalgie. Les examens cliniques cardiaque, pulmonaire et digestif étaient normaux. En raison du contexte traumatologique, une radiographie du membre supérieur gauche a été réalisée et était normale. La normalité de ces examens et la discordance entre l'anamnèse traumatologique et l'installation progressive de ce tableau de pseudoparalysie radiale périphérique distale ont motivé la réalisation d'un scanner cérébral sans puis avec injection de produit de contraste. Celui-ci a révélé de multiples plages parenchymateuses hypodenses d'œdème frontopariétales bilatérales, occipitales et temporales bilatérales. Certaines étaient centrées par des lésions arrondies spontanément isodenses et prenant le contraste en cocarde. Une lésion corticosous-corticale touchait le cortex moteur primaire de la main dans l'hémisphère droit. L'ensemble était en faveur de lésions cérébrales métastatiques, sans signe d'engagement ni atteinte méningée (Fig. 1A). La radiographie pulmonaire réalisée à la recherche d'une néoplasie primitive était normale. Le bilan sanguin (comprenant NFS, ionogramme, calcémie, bilan d'hémostase, bilan hépatique) était normal. Conformément aux résultats du scanner, l'IRM cérébrale réalisée pendant l'hospitalisation (séquences $\mathrm{T} 1$ sans puis avec injection de produit de contraste, puis avec saturation du signal de la graisse, T2 3D et FLAIR) a confirmé la présence d'une lésion dans le gyrus précentral droit correspondant à l'aire corticale motrice de la main gauche, associée à de multiples lésions intraparenchymateuses sus- et soustentorielles (Fig. 1BCDE). Il n'y avait pas d'atteinte méningée, pas d'effet de masse ni de signe d'engagement. Le scanner thoracoabdominopelvien injecté réalisé à la recherche d'une néoplasie primitive a révélé une tumeur pulmonaire du lobe supérieur gauche mesurant $29 \mathrm{~mm}$, de multiples nodules et micronodules solides arrondies des deux hémichamps pulmonaires, des adénomégalies hilaires et médiastinales suggérant un cancer pulmonaire primitif et un 




Fig. 1 Métastases cérébrales et repérage de la hand knob A à E. Imageries du patient en coupes axiales. Multiples lésions secondaires sus-tentorielles, dont l'une visible au niveau de la hand knob droite. A. Scanner cérébral avec injection : les lésions secondaires dont la lésion dans la hand knob à droite apparaissent spontanément hyperdenses, l'œdème apparaît hypodense. B. IRM séquence 3D T1 sans injection : la lésion dans la hand knob droite apparaît en hypersignal et l'œdème en hyposignal. C. IRM séquence SE-T2 : les lésions secondaires apparaissent en hyposignal, l'œdème cérébral apparaît en hypersignal et modifie les rapports normaux de la hand knob. D. IRM séquence 3D FLAIR avec injection : les lésions secondaires apparaissent en hyposignal sans hypersignal en diffusion centrale, avec des coques périphériques en hyposignal, l'œdème cérébral apparaît en hypersignal et modifie les rapports normaux de la hand knob. E. IRM séquence 3D SE T1 gadolinium avec saturation du signal de la graisse : prise de contraste des lésions secondaires avec rehaussement en cocarde $F$ et $G$. Repérage du sillon central sur une IRM cérébrale normale en séquence T1 SE. F. Dans le plan axial, le sillon central (flèches rouges) limite le lobe frontal et le lobe pariétal avec, en avant, la circonvolution frontale ascendante (cortex moteur primaire) et, en arrière, la pariétale ascendante (cortex sensitif primaire). Il est aussi reconnaissable de proche en proche en recherchant le sillon frontal supérieur (flèches noires) à la ligne interhémisphérique, en le suivant vers l'arrière jusqu'à son croisement avec le sillon précentral (tête de flèche blanche) qui est la limite antérieure de la frontale ascendante. G. Dans le plan sagittal paramédian, le sillon cingulaire (petites flèches jaunes) se poursuit en haut par sa branche marginale (tête de flèche blanche) qui limite la face postérieure de la pariétale ascendante. Le sillon central (flèche blanche) est en avant et forme une petite encoche H à L. Variations morphologiques caractéristiques du sillon central dans le plan axial sur une IRM cérébrale normale en séquence T1 [6]. La position du sillon central (flèche rouge) qui segmente la hand knob en modifie l'apparence en un oméga $(\Omega)$ inversé $(H)$, un epsilon $(\mathcal{E})$ inversé, dans sa partie latérale $(\mathrm{I})$, centrale $(\mathrm{J})$ ou médiane $(\mathrm{K})$, ou en un oméga inversé nul $(\mathrm{L})$

nodule surrénalien droit suspect de localisation secondaire. Il n'y avait pas de lésion hépatique secondaire. Un traitement prophylactique antiépileptique a été introduit. Une corticothérapie par voie intraveineuse a été débutée et a permis une régression complète du déficit neurologique de la main gauche en huit jours. Une ponction sous contrôle scanogra- phique de la tumeur pulmonaire du lobe supérieur gauche a été réalisée à $\mathrm{j} 11$ de la consultation aux urgences. L'examen anatomopathologique a conclu au diagnostic d'adénocarcinome pulmonaire différencié, avec un statut ALK négatif et EGFR négatif. Le bilan d'extension classait le cancer du patient à un stade IV (T2aN2M1c). Après concertation 
multidisciplinaire, il a été décidé de débuter une chimiothérapie associant cisplatine et pemetrexed et une irradiation encéphalique totale. L'évolution du patient fut malheureusement rapidement défavorable, son décès étant survenu au domicile à cinq semaines du diagnostic, plusieurs jours avant le début de la radiothérapie.

\section{Discussion}

Généralement, la prise en charge des patients ayant une atteinte isolée du territoire du nerf radial débute par la recherche d'une neuropathie périphérique de compression - notamment du réveil - constituant la cause la plus fréquente. D'autres diagnostics alternatifs doivent être évoqués en recherchant un traumatisme orientant vers une fracture de la diaphyse humérale, une paralysie douloureuse vers une vascularite et une cervicalgie vers une radiculopathie cervicale. L'interrogatoire doit également rechercher une intoxication au plomb dont la «pseudoparalysie de Remak » peut être inaugurale : progressive, indolore, atteignant initialement l'extenseur commun des doigts en respectant l'extenseur propre des deuxième et cinquième doigts, évoluant de façon symétrique, avec la particularité que l'activité du supinateur est conservée, d'où son appellation de "pseudoparalysie radiale ». Une pseudoparalysie radiale peut également s'intégrer dans le syndrome du défilé thoracobrachial et dans la symptomatologie de la sclérose latérale amyotrophique. Dans la pratique, un électroneuromyogramme et/ou une imagerie du rachis cervical sont souvent prescrits en première intention, parfois à défaut d'une évaluation neurologique et d'une appréciation de l'état général du patient rigoureuses, pourtant déterminantes tant elles modifient la stratégie de prise en charge. Dans la littérature neurologique, un point clinique est essentiel : l'absence de déficit sensitif associé à une symptomatologie distale motrice du nerf radial est un signe d'alerte en faveur d'une cause centrale [1-3].

Au niveau anatomique, l'aire motrice de la main - dite hand knob - se situe dans le cortex précentral (cortex moteur primaire, aire 4 de Brodmann) au niveau du sillon central. Il se situe à la limite entre le lobe frontal et le lobe pariétal avec, en avant, la circonvolution frontale ascendante (cortex moteur primaire M1) et, en arrière, la circonvolution pariétale ascendante (cortex sensitif primaire) $[1,2,4,5]$ (Fig. 1FG). À l'IRM dans le plan axial, cinq variantes morphologiques typiques du sillon central sont décrites [6] (Fig. 1HIJKL). Il est reconnaissable par sa forme en oméga ou en epsilon, inversés par le relief de la hand knob $[4,6]$. Certains auteurs suggèrent que la portion médiale de la hand knob correspond à la topographie ulnaire des doigts et qu'elle est davantage atteinte par des mécanismes de bas débit cérébral ; et que la partie latérale de la hand knob correspond à la topographie radiale des doigts, davantage atteinte par des mécanismes cardioemboliques [7]. Une lésion du lobe pariétal inférieur ou du gyrus angulaire inférieur peut aussi entraîner un déficit moteur de la main, de la préhension, de la manipulation et des mouvements précis des doigts, avec une possible atteinte associée de la sensibilité complexe $[1,8]$.

En l'absence de cause périphérique évidente, la littérature neurologique insiste sur la nécessité de rechercher et de traiter comme telle une atteinte cérébrale ischémique en réalisant une IRM cérébrale. Les accidents ischémiques de la hand knob dits rare stroke syndromes y apparaissent comme l'étiologie centrale quasi exclusive d'un déficit moteur isolé de la main. Leur incidence parmi les accidents ischémiques cérébraux est estimée entre 0,7 et $1,5 \%[1,7,9]$. Une cause centrale plus rare encore de pseudoparalysie radiale est le glioblastome dont l'incidence parmi les tumeurs cérébrales varie entre 16 et $23 \%$ selon les séries [10]. L'incidence des tumeurs cérébrales primitives touchant l'aire corticale motrice de la main n'est pas connue [10]. Enfin, une seule publication rapporte un cas de métastases cérébrales localisées dans la hand knob et révélées par une pseudoparalysie radiale isolée de la main, avec un diagnostic final d'adénocarcinome pulmonaire. L'issue avait également été rapidement fatale pour le patient [11]. Les métastases cérébrales représentent la première cause des tumeurs intracrâniennes. Elles sont majoritairement de localisation sus-tentorielle (80\%), mais l'épidémiologie des métastases touchant la hand knob n'est pas décrite [12]. Leur présentation initiale mime rarement un accident vasculaire cérébral (moins de $3 \%$ des cas) ; les céphalées ( $50 \%$ ), la crise convulsive, le plus souvent focale (20 à $40 \%$ ), étant les modes de révélation les plus fréquents $[13,14]$. Si tous les cancers peuvent métastaser au niveau cérébral, la découverte de lésions cérébrales suspectes de malignité impose de rechercher par ordre de fréquence : un cancer bronchopulmonaire ( $40 \%$ des cas), un cancer du sein (20\%) et un mélanome (5\%) [14]. En l'absence de traitement rapide et agressif, la durée médiane de survie de ces patients est inférieure à trois mois. Les mutations EGFR et ALK - absentes chez ce patient - sont connues comme des facteurs de bon pronostic pour les adénocarcinomes pulmonaires métastatiques au niveau cérébral [14]. Dans notre cas, le score pronostique développé par Rades et al. intégrant l'intervalle entre le diagnostic initial et l'irradiation encéphalique totale, l'âge, l'indice de Karnofsky, et la présence de métastases extracérébrales a donné une estimation réaliste du pronostic du patient avec une durée attendue de survie de 1,3 mois [15].

En conclusion ce cas confirme l'importance de ne pas méconnaître le tableau de pseudoparalysie radiale avant de conclure à une hypothétique cause classique de paralysie radiale périphérique. Il ajoute que les atteintes de la hand $k n o b$ peuvent être causées non seulement par un accident ischémique cérébral, mais aussi par une métastase cérébrale 
symptomatique. Le scanner étant moins performant pour l'étude de la hand knob, l'IRM constitue l'examen de référence pour explorer tout déficit moteur isolé de la main sans explication évidente et rapide pour une cause périphérique.

Liens d'intérêts : les auteurs déclarent ne pas avoir de liens d'intérêts.

\section{Références}

1. Alstadhaug KB, Sjulstad A (2013) Isolated hand paresis: a case series. Cerebrovasc Dis Extra 3:65-73

2. Celebisoy M, Ozdemirkiran T, Tokucoglu F, et al (2007) Isolated hand palsy due to cortical infarction: localization of the motor hand area. Neurologist 13:376-9

3. Chen PL, Hsu HY, Wang PY (2006) Isolated hand weakness in cortical infarctions. J Formos Med Assoc 105:861-5

4. Krainik A, Feydy A, Colombani JM, et al (2003) Functional anatomy of the central nervous system. J Radiol 84:285-97

5. Yousry TA, Schmid UD, Alkadhi H, et al (1997) Localization of the motor hand area to a knob on the precentral gyrus. A new landmark. Brain 120:141-57
6. Peters N, Müller-Schunk S, Freilinger T, et al (2009) Ischemic stroke of the cortical hand knob area: stroke mechanisms and prognosis. J Neurol 256:1146-51

7. Kim JS (2001) Predominant involvement of a particular group of fingers due to small cortical infarction. Neurology 56:1677-82

8. Orosz P, Szőcs I, Rudas G, et al (2018) Cortical hand knob stroke: report of 25 cases. J Stroke Cerebrovasc Dis 27:1949-55

9. Timsit S, Logak M, Manai R, et al (1997) Evolving isolated hand palsy: a parietal lobe syndrome associated with carotid artery disease. Brain 120:2251-7

10. Fang S, Liang J, Qian T, et al (2017) Anatomic location of tumor predicts the accuracy of motor function localization in diffuse lower-grade gliomas involving the hand knob area. AJNR 38:1990-7

11. Folyovich A, Varga V, Várallyay G, et al (2018) A case report of isolated distal upper extremity weakness due to cerebral metastasis involving the hand knob area. BMC Cancer 18:947

12. Delattre JY, Krol G, Thaler HT, et al (1988) Distribution of brain metastases. Arch Neurol 45:741-4

13. Morgenstern LB, Frankowski RF (1999) Brain tumor masquerading as stroke. J Neurooncol 44:47-52

14. Taillibert S, Le Rhun E (2015) Épidémiologie des lésions métastatiques cérébrales. Cancer/Radiothérapie 19:3-9

15. Rades D, Dunst J, Schild SE (2008) A new scoring system to predicting the survival of patients treated with whole-brain radiotherapy for brain metastases. Strahlenther Onkol 184:251-5 